\title{
Geometrical Similarity Criterion for Electromagnetic Drives Magnetic Systems with respect to Permissible Heating Condition
}

\author{
V.Yu. Neyman \\ Novosibirsk State Technical University \\ Novosibirsk, Russian Federation \\ e-mail: nv.nstu@ngs.ru
}

\author{
L.A Neyman \\ Novosibirsk State Technical University \\ Novosibirsk, Russian Federation \\ e-mail: neyman31@gmal.com
}

\author{
A.S. Shabanov \\ Novosibirsk State Technical University \\ Novosibirsk, Russian Federation \\ e-mail: as_schabanov@mail.
}

\begin{abstract}
The paper presents the results of investigation of geometrically similar magnetic cores of electromagnetic drives magnetic systems with respect to heating conditions. As a result, the geometrical similarity criterion has been obtained. This criterion is a non-linear function of cooling surface and electromagnet volume. It simplifies design optimization procedures when volumes are different in typical variants of magnetic systems. The geometrical similarity criterion has been tested by means of magnetic field simulation.
\end{abstract}

Keywords-electromagnetic drive; magnetic system; heat similarity factor; geometrical similarity criterion; electromagnet volume.

\section{INTRODUCTION}

Impact electromagnetic machines and devices are widely used in industry for mechanization of many technological processes [1-6]. Electromagnetic machines design methods are permanently improved [7-15].

The paper states the results of electromagnetic drives magnetic systems investigation. The purpose of the research is to establish an influence of geometrically similar electromagnet cores volume on design factor with respect to core linear dimensions dependence on its volume.

\section{LINEAR DIMENSIONS CRITERION}

The base expression accepted as a measure of linear dimensions for geometrically similar electromagnet cores in operating conditions [16] is:

$$
K_{\mathrm{T}}=\frac{P}{k_{\mathrm{T}} \mathrm{\phi} \sqrt[3]{V^{2}}}
$$

where $K_{\mathrm{T}}$ is the heat similarity factor, $P$ is the consumed power, $k_{\mathrm{T}}$ is the heat-transfer factor, $\phi$ is the temperature excess, $V$ is the electromagnet volume.

The equation (1) is chosen to be the main criterion as electromagnetic unit reliability depends on degree of winding temperature excess stability in recursive short-time and longtime modes. Evidently there is no explicit linear dimension volume dependency in (1) for geometrically similar systems.

As decrease of dimensions effects on the windings temperature excess stability, it is important to obtain a relationship connecting volume, heat dissipation areas and linear dimensions of geometrically similar electromagnets.

In DC systems, core loss is negligible; then power dissipation is:

$$
P=I^{2} R,
$$

where $I$ is the current in the winding, $R$ is the winding wire resistance, which is defined as:

$$
R=\mathrm{c} \frac{l_{\mathrm{av}} w}{q}=\mathrm{c} \frac{\pi d_{\mathrm{av}} w}{q}=\mathrm{c} \frac{\mathrm{p}\left(d_{1}+d_{2}\right) w}{2 q},
$$

where $\mathrm{c}$ is the wire specific resistance, $l_{\mathrm{av}}$ is the turn average length, $d_{\mathrm{av}}$ is the turn average diameter, $d_{1}$ is the winding internal diameter, $d_{2}$ is the winding external diameter, $w$ is the number of turns, $q$ is the wire cross-section, which is defined as:

$$
q=\frac{S_{\mathrm{ap}} k_{\mathrm{or}}}{w}=\frac{h_{\mathrm{ap}} l_{\mathrm{w}} k_{\mathrm{or}}}{w}
$$

where $S_{\mathrm{ap}}$ is the winding aperture cross-section area, $h_{\mathrm{ap}}$ is the aperture height, $l_{\mathrm{w}}$ is the winding length, $k_{\mathrm{or}}$ is the 
aperture occupation ratio.

Then, from (1)-(4) the expression for magnetic force is:

$$
I w=\sqrt{\frac{K_{\mathrm{T}} 2 k_{\mathrm{T}} \phi S_{\mathrm{ap}} k_{\mathrm{or}}}{\mathrm{p}\left(d_{1}+d_{2}\right) \mathrm{c}} \sqrt[3]{V^{2}}} .
$$

The magnetic force equation for the prolonged heating mode can be derived directly from the Newton heat-balance equation:

$$
P=k_{\mathrm{T}} S_{\mathrm{c}} \phi
$$

where $S_{\mathrm{c}}$ is the winding cooling surface area.

If the heat is sunk only from external and internal surfaces of the winding:

$$
S_{\mathrm{c}}=\mathrm{p}\left(d_{1}+d_{2}\right) l_{\mathrm{w}},
$$

then magnetic force is obtained from (6) with respect to (2) (4), (7):

$$
I w=\sqrt{\frac{2 k_{\mathrm{T}} \phi S_{\mathrm{ap}} k_{\mathrm{or}} l_{\mathrm{w}}}{\mathrm{c}}} .
$$

Let us note that (5) and (8) are equivalent for all systems where heating is caused only by losses in the winding. These expressions of magnetic force describe the heating mode when winding overheating temperature value is no more than allowable one.

Equation (5) can be represented as:

$$
I w=\sqrt{\frac{K_{\mathrm{T}} \sqrt[3]{V^{2}}}{\mathrm{p}\left(d_{1}+d_{2}\right) l_{\mathrm{w}}}} \sqrt{\frac{2 k_{\mathrm{T}} \phi S_{\mathrm{w}} k_{\mathrm{or}} l_{\mathrm{w}}}{\mathrm{c}}} .
$$

Equivalence of (8) and (9) gives:

$$
\frac{K_{\mathrm{T}} \sqrt[3]{V^{2}}}{\mathrm{p}\left(d_{1}+d_{2}\right) l_{\mathrm{w}}}=1
$$

With respect to (7) equation (10) gives the expression involving only geometrical parameters:

$$
K_{\mathrm{T}}=\frac{S_{\mathrm{c}}}{\sqrt[3]{V^{2}}}=\text { const }
$$

Thus, the identical relation (11) establishes the system heat exchanging surface area - volume ratio.

\section{COMPARISION OF DC ELECTROMAGNETS WITH GEOMETRICALLY SIMILAR CORES}

To estimate the heat similarity factor it is necessary to compare DC electromagnets geometrically similar magnetic cores.

Validity of the equation (11) for all geometrically similar systems is tested for the typical cylinder electromagnet design (Fig. 1).(5) The electromagnet dimensions are $d_{1}=120 \mathrm{~mm}, d_{2}=218 \mathrm{~mm}, l_{w}=273 \mathrm{~mm}$.

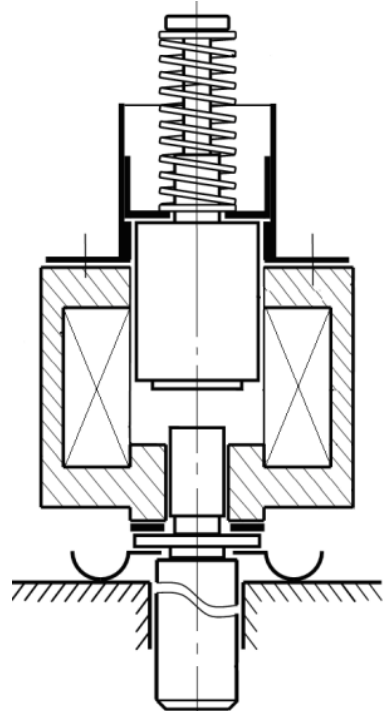

Fig. 1. Cylinder electromagnet design

The basic design data including the heat similarity factor (11) for electromagnets models are presented for several geometrically similar systems in Table I.

TABLE I. MAGNETIC PROPERTIES VALUES

\begin{tabular}{|l|c|c|c|c|c|c|}
\hline $\begin{array}{l}\text { Model } \\
\text { volume, } \\
\mathrm{m}^{3}\end{array}$ & $V$ & $2 V$ & $4 V$ & $6 V$ & $8 V$ & $10 V$ \\
\cline { 2 - 7 } & 0.0204 & 0.0409 & 0.0818 & 0.1226 & 0.1635 & 0.2044 \\
\hline$S_{\mathrm{c}}, \mathrm{m}^{2}$ & 0.1484 & 0.469 & 0.746 & 0.977 & 1.184 & 1.374 \\
\hline$K_{\mathrm{T}}$ & 3.96 & 3.96 & 3.96 & 3.96 & 3.96 & 3.96 \\
\hline
\end{tabular}

Linear dimension scale factor is calculated as:

$$
M=\sqrt[3]{m_{\mathrm{V}}}
$$

where $m_{\mathrm{V}}$ is the volume zoom ratio.

As it follows from Table I the geometrically similar cylindrical electromagnets have the same heat similarity factor $K_{T}=3.96$. The proposed geometrical similarity criterion is valid for other magnetic cores of geometrically similar DC electromagnets. It has different absolute values for different types of geometrically similar electromagnets. 
Therefore, (11) can be recommended as a valid geometrical criterion of "thermal similarity" to compare electromagnets of any type. Design factor-volume ratios are obtained at the same windings average overheating temperature $\phi=100^{\circ} \mathrm{C}$.

Magnetic forces in windings for the long-time mode were calculated by (8) with respect to the electromagnet design in Fig. 1, the number of turns $w=1800$ and the steel saturation curve in Fig. 2.

Then investigation of geometrically similar electromagnets gave the efficiency factor $(E)$ - design factor $(D)$ ratio at the overheating temperature $\phi=100^{\circ} C$. It was found that maximal design factor values are in the range from 380 to 455 $\sqrt{\mathrm{N}} / \mathrm{m}^{2}$ (Fig. 3) for volume zoom ratios from 0.125 to $16.0 \mathrm{in}$ Table II.

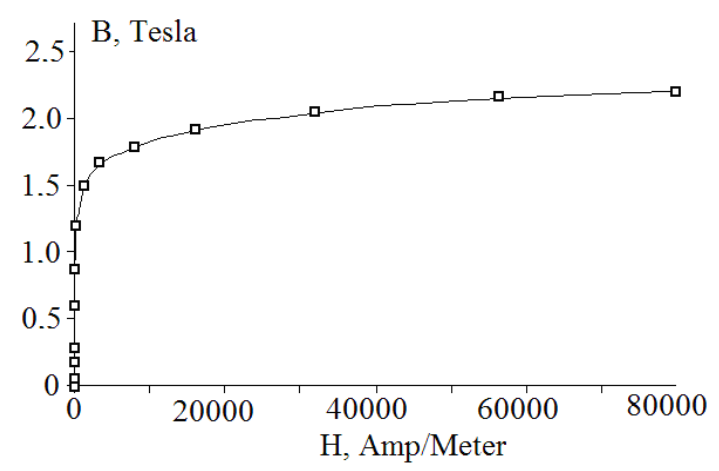

Fig. 2. Steel saturation curve

Electromagnet volume was varied keeping complete geometrical similarity. Linear dimensions dependence on volume change is expressed with dimensionless scaling factor

$$
M=\sqrt[3]{k_{\mathrm{V}}}
$$

where $k_{\mathrm{V}}$ is the volume zoom ratio $0<k_{\mathrm{V}}<\infty$.

Simulation parameters are stated in Table II.

TABLE II. SIMULATION PARAMETERS

\begin{tabular}{|c|cc|c|c|c|c|}
\hline$k_{\mathrm{V}}$ & \multicolumn{2}{|c|}{} & 0.125 & 0.25 & 0.5 & 1,0 \\
\hline$V_{\mathrm{cu}}$ & $10^{-4}$ & $\mathrm{~m}^{3}$ & 8.4 & 16.8 & 33.5 & 66.8 \\
\hline$V_{\mathrm{st}}$ & $10^{-4}$ & $\mathrm{~m}^{3}$ & 17.2 & 34.7 & 69.3 & 137.6 \\
\hline$I w$ & \multicolumn{2}{|c|}{$\mathrm{A}$} & 5670 & 8321 & 11160 & 16650 \\
\hline$k_{\mathrm{V}}$ & \multicolumn{2}{|c|}{-} & 2.0 & 4.0 & 8.0 & 16.0 \\
\hline$V_{\mathrm{cu}}$ & $10^{-4}$ & $\mathrm{~m}^{3}$ & 134.1 & 267.8 & 540.0 & 1071.9 \\
\hline$V_{\mathrm{st}}$ & $10^{-4}$ & $\mathrm{~m}^{3}$ & 276.9 & 552.6 & 1115.3 & 2211.8 \\
\hline$I w$ & \multicolumn{2}{|c|}{$\mathrm{A}$} & 23400 & 33159 & 47340 & 62710 \\
\hline
\end{tabular}

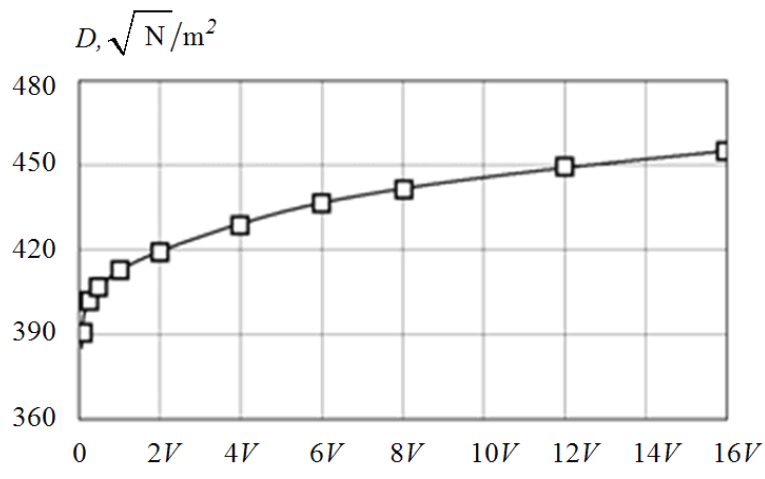

Fig. 3. Electromagnet design factor - volume ratio

As it is shown in Fig. 4, the electromagnet volume change strongly effects on the design and efficiency factors.

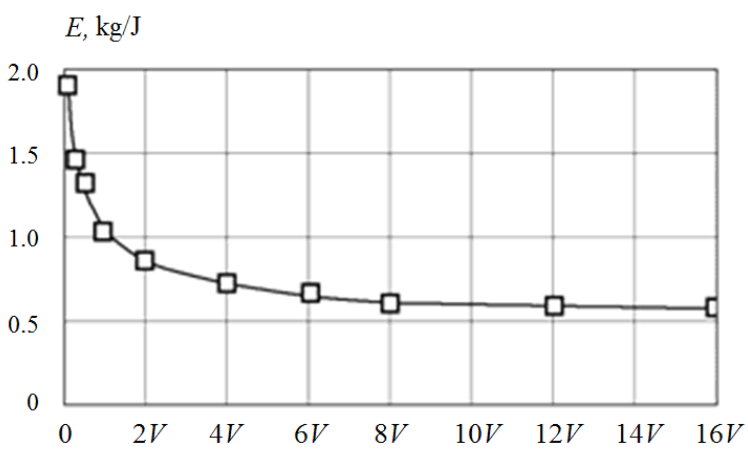

Fig. 4. Electromagnet efficiency factor-volume ratio

Volume increase leads to a decrease of material consumption and, after all, reducing of electromagnetic forces work per volume unit. It follows from Fig. 4 that volume increase causes less efficiency factor growth and its stabilization at $E=0.5$.

\section{CONCLUSION}

The design factor-volume function has been obtained for the typical construction of the cylindrical electromagnet. It helps to estimate the influence of geometrical similarity of magnetic cores volume values on design factor.

Therefore, it is necessary to develop a method to consider the design factor more precisely with respect to electromagnet construction type and dimensions.

\section{References}

[1] N.P. Ryashentsev, E.M. Timoshenko, A.V. Frolov, "Theory, calculation and design of impact electromagnetic machines", Novosibirsk: Science, Siberian Branch, p. 260, 1970.

[2] N.P. Ryashentsev, G.G. Ugarov, A.V. Lvitsin, "Electromagnetic presses", Novosibirsk: Science, Siberian Branch, p. 216, 1989. 
[3] A.T. Malov, N.P. Ryashentsev and others, "Electromagnetic hammers", Novosibirsk: Science, p. 128, 1968.

[4] N.P. Ryashentsev, V.N. Ryashentsev, "Electromagnetic drive of linear machines", Novosibirsk: Science, p. 153, 1985.

[5] B.F. Simonov, V.Y. Neyman, A.S. Shabanov, "New conception of an electromagnetic drive for a vibration source in hole", The 18 international conference of young specialists on micro/nanotechnologies and electron devices, EDM 2017: proc., Altai, Erlagol, 29 June - 3 July 2017, p. 507-510, 2017.

[6] K.M. Usanov, A.V. Volgin, E.A. Chetverikov, V.A. Kargin, A.P. Moiseev, Z.I. Ivanova, "Strike action electromagnetic machine for immersion of rod elements into ground", IOP Conference Series: Earth and Environmental Science", [Electronic resource], p. 032050, 2017.

[7] D.V. Batischev, A.V. Pavlenko, "On designing electromagnetic drives operating under conditions of high vibrations", Russian Electrical Engineering, vol. 83, Num. 8, p. 423, 2012.

[8] V.I. Moshkin, D.N. Shestakov, S.Y. Pomyalov, G.G. Ugarov, "Mathematical simulation of electromagnetic pulse linear motors", International Conference on Actual Problems of Electron Devices Engineering, APEDE 2014, pp. 348-352, 2014.

[9] Y.I. Klimenko, D.V. Batishchev, A.V. Pavlenko, V.P. Grinchenkov, "Design of a linear electromechanical actuator with an active vehicle suspension system", Russian Electrical Engineering, vol. 86, Num. 10, pp. 588-593, 2015.

[10] R.R. Sattarov, "Electromechanical transients in passive suspension systems with eddy current dampers" 9th International Conference on
Power Drives Systems, ICPDS 2016, Conference Proceedings 9, p. 7756676, 2016.

[11] L.A. Neyman, V.Y. Neyman, K.A. Obukhov, "New method of the synchronous vibratory electromagnetic machine mechatronic module control" The 18 international conference of young specialists on micro/nanotechnologies and electron devices, EDM 2017: proc., Altai, Erlagol, 29 June - 3 July 2017, pp. 516-519, 2017.

[12] L.A. Neyman, V.Y. Neyman, "Dynamic model of a vibratory electromechanical system with spring linkage" 11 International forum on strategic technology (IFOST 2016): proc., Novosibirsk, 1-3 June 2016, Pt. 2, pp. 23-27, 2016.

[13] V.P. Pevchev, "Science of mining machines the superexcitation and efficiency relation in a short-stroke pulsed electromagnetic motor of a seismic source", Journal of Mining Science, vol. 46, Num. 6, pp. 656$665,2010$.

[14] Y.M. Zaitsev, O.A. Petrov, N.V. Russova, G.P. Svintsov, I.P. Ivanov, A.V. Prikazshchikov, "Minimizing the power consumption of a clappertype dc electromagnet in intermittent operation", Russian Electrical Engineering, vol. 86, Num. 8, pp. 474-478, 2015.

[15] A.A. Tatevosyan, A.S. Tatevosyan, "Calculation of magnetic system of the magnetoelectric machines", Dynamics of Systems, Mechanisms and Machines, Dynamics 2014 Proceedings, p. 7005698, 2014.

[16] G.A. Bugayev, "Electromagnets estimation criterions," Electricity, Num. 11, pp. 51-55, 1966. 